\title{
Numerical solution of the Gross-Pitaevskii equation using an explicit finite-difference scheme: An application to trapped Bose-Einstein condensates
}

\author{
M. M. Cerimele, ${ }^{1}$ M. L. Chiofalo, ${ }^{2}$ F. Pistella, ${ }^{1}$ S. Succi, ${ }^{1}$ and M. P. Tosi ${ }^{2}$ \\ ${ }^{1}$ Istituto Applicazioni Calcolo “M. Picone," Via del Policlinico 137, I-00161 Roma, Italy \\ ${ }^{2}$ INFM and Classe di Scienze, Scuola Normale Superiore, Piazza dei Cavalieri 7, I-56126 Pisa, Italy
}

(Received 21 May 1999; revised manuscript received 17 February 2000)

\begin{abstract}
We present the application of a fast, explicit time-marching scheme for the solution of the Gross-Pitaevskii equation in cylindrical geometry. The scheme is validated on simple analytical tests and demonstrated for two situations of physical interest in experiments on the Bose-Einstein condensation (BEC) of trapped alkali-metal vapors. It is tested by reproducing known results on the free expansion of a BEC after removing a cylindrical trap, and it is then used to address the formation of matter-wave pulses that result from gravity-induced transport of a condensate in an optical potential.
\end{abstract}

PACS number(s): 02.70.-c, 03.75.Fi

\section{INTRODUCTION}

The observation of the Bose-Einstein condensation (BEC) of trapped atomic vapors of ${ }^{87} \mathrm{Rb},{ }^{23} \mathrm{Na}$, and ${ }^{7} \mathrm{Li}[1-3]$ has spurred great excitement in the atomic physics community and a renewed interest in the study of the collective dynamics of macroscopic assemblies of atoms in the same quantum state $[4,5]$. This state of matter, whose existence was predicted back in the 1920s, exhibits several characteristics that set it apart from other condensed-matter systems [6]. In fact, besides internal interactions, the macroscopic behavior of BEC matter is highly sensitive to external conditions, and primarily to the shape of the external trapping potential.

Trapped condensates of alkali-metal atoms are easily accessible to theoretical predictions since the interactions are effectively modeled by a single parameter (the scattering length), and the external potentials are described by a few parameters that are accurately known from experiment. The progress on the experimental side is stimulating a corresponding wave of activity on both theoretical and numerical fronts. The present paper is a contribution to the latter.

\section{IMPLICIT VERSUS EXPLICIT SCHEMES FOR BEC PROBLEMS}

To date, the numerical simulation of the time-dependent Gross-Pitaevskii equation (see below) describing the zerotemperature mean-field dynamics of a dilute BEC has been handled mostly by implicit time-marching techniques $[7,8]$. The main merit of the implicit approach is to march in large time steps to the steady state without any stability constraint. The price for stability is the need to solve a linear algebraic system at each time step, which is a rather expensive computational task. In addition, although harmless to stability, the time-step size must nonetheless be carefully watched for reasons that regard the numerical accuracy of the time evolution. For steady-state problems, the latter is no concern, and implicit methods are certainly the method of choice.

Explicit methods have precisely opposite virtues and drawbacks. They are stability bound, but much faster on a per-time-step basis. In addition, since the time step is severely constrained anyway, they generally tend to produce time-accurate solutions. The general feeling is that explicit methods are the methods of choice for time-dependent problems with a rich, fast-moving dynamics. This seems indeed the case of Bose-Einstein condensates. In light of the above, there appears to be a wide scope for exploring the applicability of explicit methods to problems in the area of BoseEinstein condensation.

\section{GROSS-PITAEVSKII EQUATION}

The Bose-Einstein condensation of a dilute quantum gas is a complex many-body problem, whose complete description would in principle require a fully quantum kinetic treatment [4]. However, the zero-temperature dynamics of a finite, dilute system of weakly interacting bosons is well captured by a mean-field approach whereby the condensate is described by a single global wave function $\Psi(\mathbf{r}, \mathbf{t})$. The evolution of the condensate wave function in an external potential $V_{\text {ext }}(\mathbf{r})$ is described by the Gross-Pitaevskii equation (GPE) $[9,10]$ :

$$
i \hbar \partial_{t} \Psi=\left[-\frac{\hbar^{2}}{2 m} \Delta+V_{\text {ext }}(\mathbf{r})+N U_{0}|\Psi|^{2}\right] \Psi .
$$

Here, $N$ is the number of bosons, $U_{0} \equiv 4 \pi \hbar^{2} a / m$ is the selfinteraction coupling, and $a$ is the boson-boson $s$-wave scattering length. In the earlier experiments, the external potential is typically in the form of a harmonic well, $V_{\text {ext }}(\mathbf{r})$ $=\frac{1}{2} m \omega^{2}\left(x^{2}+y^{2}+\epsilon z^{2}\right)$, where $\omega$ is the frequency and $\epsilon$ the aspect ratio of the (cylindrical) trap. More recently, condensed vapors of ${ }^{87} \mathrm{Rb}$ atoms have been confined in a vertical lattice of optical traps under the influence of gravity [11], the external potential being of the form $V_{\text {ext }}(\mathbf{r})$ $=V_{0} e^{-\kappa^{2} r^{2}} \sin ^{2}(2 \pi z / d)-m g z$ with $\kappa$ a characteristic inverse trapping length and $d$ the lattice constant.

The numerical prediction of time-dependent BEC dynamics within the mean-field GPE picture presents a significant computational challenge. To this end, a number of techniques have been proposed in the recent past, ranging from implicit alternating-direction-implicit solvers [7] to customized versions of linear eigenvalue solvers [12] and functional minimization techniques for the time-independent version of 
Eq. (1) [13]. In this paper, we present an alternative method to solve the time-dependent GPE using an explicit timemarching technique. The method is an extension to the BEC context of a fast explicit, time-staggered scheme proposed by Visscher [14] to solve the Schrödinger equation in an external potential. This extension is nontrivial on account of (i) the cylindrical geometry, which requires careful near-axis treatment, and (ii) the nonlinearity associated with the selfinteraction potential. In fact, even though the theoretical and numerical apparatus of Visscher's scheme carries over naturally to the GPE framework, the physical richness and complexities associated with the nonlinear potential can only be assessed by actual numerical experimentation.

\section{NUMERICAL SCHEME}

Equation (1) is made dimensionless by adopting the scale units

$$
S_{l}=\sqrt{\hbar / 2 m \omega}, \quad S_{t}=1 / \omega, \quad S_{E}=\hbar \omega
$$

for length, time, and energy, respectively. Upon rescaling the wave function by a factor $\rho$, the dimensionless radial coordinate, we obtain the dimensionless form of the GPE:

$$
i \frac{\partial}{\partial \tau} \Phi=[\mathcal{T}+\mathcal{V}] \Phi
$$

where

$$
\begin{gathered}
\Phi=\rho\left(\Psi S_{l}^{3 / 2}\right), \\
\mathcal{T}=-\frac{\partial^{2}}{\partial^{2} \zeta^{2}}-\frac{\partial^{2}}{\partial^{2} \rho^{2}}+\frac{1}{\rho} \frac{\partial}{\partial \rho}-\frac{1}{\rho^{2}}, \\
V=\frac{1}{4}\left(\rho^{2}+\epsilon \zeta^{2}\right)+\frac{8 \pi N \alpha}{\rho^{2}}|\Phi|^{2},
\end{gathered}
$$

and $\rho \equiv r / S_{l}, \alpha \equiv a / S_{l}, \zeta \equiv z / S_{l}$, and $\tau \equiv \omega t$.

Centered differencing is the natural approach to the approximation of the space derivatives appearing in the kinetic energy operator $\mathcal{T}$. As to the approximation of the left-hand side of Eq. (3), some care is necessary. As shown by Visscher for the one-dimensional case, an explicit approach requires the definition of a staggered time grid to avoid numerical instability and to assure the conservation of the wave function density. More specifically, the real and imaginary parts of $\Phi$ are computed at even and odd time steps, respectively, according to the following scheme:

$$
\begin{gathered}
\operatorname{Re}\left(\Phi_{l, j}^{2 k}\right)=\operatorname{Re}\left(\Phi_{l, j}^{2 k-2}\right)+2 \Delta \tau\left(\mathcal{T}+\mathcal{V}^{2 k-1}\right) \operatorname{Im}\left(\Phi_{l, j}^{2 k-1}\right), \\
\operatorname{Im}\left(\Phi_{l, j}^{2 k+1}\right)=\operatorname{Im}\left(\Phi_{l, j}^{2 k-1}\right)-2 \Delta \tau\left(\mathcal{T}+\mathcal{V}^{2 k}\right) \operatorname{Re}\left(\Phi_{l, j}^{2 k}\right),
\end{gathered}
$$

where $\Phi_{l, j}^{k} \equiv \Phi(l \Delta \rho, j \Delta \zeta ; k \Delta \tau)$ and $\operatorname{Re}\left(\Phi_{l, j}^{0}\right)$ and $\operatorname{Im}\left(\Phi_{l, j}^{1}\right)$ are supposed to be assigned at $\tau=0$. This time-staggered procedure is patterned after trajectory integration of Hamiltonian dynamics, as suggested by the Hamiltonian structure of the Schrödinger equation $d \mathrm{Re} / d t=\mathcal{H} \operatorname{Im}$ and $d \operatorname{Im} / d t=-\mathcal{H} \operatorname{Re}$,
$\mathrm{Re}$, and $\mathrm{Im}$ being the real and imaginary parts of the wave function and $\mathcal{H}=\mathcal{T}+\mathcal{V}$ the Hamiltonian operator.

The proper definition of the probability density $P=\mathrm{Re}^{2}$ $+\mathrm{Im}^{2}$ is not obvious in the numerical scheme since the real and imaginary components are staggered, i.e., defined at alternate times. However, as shown by Visscher [14], either choice $P_{2 k}=\operatorname{Re}_{2 k}^{2}+\operatorname{Im}_{2 k-1} \operatorname{Im}_{2 k+1}$ or choice $P_{2 k+1}$ $=\operatorname{Re}_{2 k} \operatorname{Re}_{2 k+2}+\operatorname{Im}_{2 k+1}^{2}$ ensures probability conservation with a time-independent potential.

Since our Hamiltonian does depend on time via the nonlinear self-interaction term, probability conservation has to be checked carefully. To this purpose, and also for computational convenience, we turn to a slight modification of the Visscher scheme whereby both real and imaginary parts of the wave function are synchronized on the same series of discrete time instants. For convenience, we shall refer to this version as the synchronous Visscher scheme.

\section{A. Synchronous Visscher scheme}

We analyze a simplified variant of the Visscher scheme, based on the idea of nonstaggered explicit time marching. The idea is to advance both real/imaginary components in units of two time steps using the intermediate, centered value of the imaginary/real component. Namely,

$$
\begin{aligned}
& \operatorname{Re}\left(\Phi_{l, j}^{k+1}\right) \equiv \operatorname{Re}\left(\Phi_{l, j}^{k-1}\right)+2 \Delta \tau\left(\mathcal{T}+\mathcal{V}^{k}\right) \operatorname{Re}\left(\Phi_{l, j}^{k}\right), \\
& \operatorname{Im}\left(\Phi_{l, j}^{k+1}\right)=\operatorname{Re}\left(\Phi_{l, j}^{k-1}\right)-2 \Delta \tau\left(\mathcal{T}+\mathcal{V}^{k}\right) \operatorname{Re}\left(\Phi_{l, j}^{k}\right) .
\end{aligned}
$$

We shall now provide a few details on the following aspects of the synchronous scheme, namely: (i) start-up procedure, (ii) unitarity (norm conservation), and (iii) stability. Before doing so, it is worth emphasizing that the synchronous Visscher scheme is very similar to second-order difference methods proposed by Kosloff [15] for the timedependent Schrödinger equation. To the best of our knowledge, this is the first time such a scheme has been applied to the Gross-Pitaevskii equation in the context of BEC problems.

\section{Start-up procedure}

The scheme is initiated with a single Euler-forward step [17] from $k=0$ to $k=1$ using the above equation (6) with a time step $\Delta \tau$ :

$$
\begin{gathered}
\operatorname{Re}\left(\Phi_{l, j}^{1}\right)=\Delta \tau\left(\mathcal{T}+\mathcal{V}^{0}\right) \operatorname{Im}\left(\Phi_{l, j}^{0}\right), \\
\operatorname{Im}\left(\Phi_{l, j}^{1}\right)=-\Delta \tau\left(\mathcal{T}+\mathcal{V}^{0}\right) \operatorname{Re}\left(\Phi_{l, j}^{0}\right) .
\end{gathered}
$$

Once level $k=1$ is available, the time marching can proceed in steps of $2 \tau$ as indicated in Eq. (6).

\section{Unitarity}

As anticipated, due to the nonlinear nature of the GrossPitaevskii equation, it is crucial to show that the present scheme still preserves unitarity at each discrete time $t_{k}$. By defining the probability density at the discrete space location $(l, j)$ and discrete time $k+1$ as follows:

$$
P_{l, j}^{k+1} \equiv \operatorname{Re}_{l, j}^{k+1} \operatorname{Re}_{l, j}^{k}+\operatorname{Im}_{l, j}^{k+1} \operatorname{Im}_{l, j}^{k},
$$


it is readily shown that the change in time of the overall probability integrated over the entire computational domain is indeed zero:

$$
\sum_{l, j}\left(P_{l, j}^{k+1}-P_{l, j}^{k}\right)=0,
$$

provided boundary conditions are such that they annihilate surface terms. This is indeed the case with our applications, since we impose a vanishing wave function on the outer boundary $\rho=\rho_{\max }$ and periodicity along $\zeta$. The proof of unitarity is given in the Appendix.

\section{Stability}

As for any explicit scheme, the main question is to assess the largest time step that can be used without compromising numerical stability. Standard analysis of the spectrum of the discretized evolution operator [16] shows that the stability limit is

$$
\Delta \tau\left(\left|4 / \Delta^{2}+V_{M}\right|\right)<2 .
$$

Here, $\Delta^{-2} \equiv \Delta_{\rho}^{-2}+\Delta_{\zeta}^{-2}$ where $\Delta_{\rho}$ and $\Delta_{\zeta}$ are the mesh spacings along the radial and axial directions and $V_{M}$ is the maximum value of the potential field.

The above relation identifies the largest acceptable time step $\Delta \tau_{c}$ as

$$
\Delta \tau_{c}=\frac{\Delta^{2} / 2}{1+V_{M} \Delta^{2} / 4} .
$$

This expression shows that interaction potentials below the numerical threshold $V_{M}<V_{\Delta} \equiv 4 / \Delta^{2}$ do not affect the standard Courant-Friedrichs-Lewy condition $\Delta \tau \sim \Delta^{2}$ for the diffusion equation. This is a severe bound since it forces the time step to decrease with the square of the grid spacing.

Above $V_{\Delta}$, the time step is basically controlled by the potential alone, $\Delta \tau<1 / V_{M}$, independently of the mesh size. This regime implies certain limitations on the physical parameters characterizing the interaction potential and in particular the maximum number of bosons allowed in the numerical simulation.

Let us inspect the condition $V<V_{\Delta}$ for the case of the self-interacting potential; that is, $8 \pi \alpha N S_{l}^{3}|\Psi|^{2}<4 / \Delta^{2}$. By taking $S_{l}^{3}|\Psi|^{2} \sim\left(\pi N_{\rho}^{2} N_{\zeta} \Delta_{\rho}^{2} \Delta_{\zeta}\right)^{-1}$ we obtain

$$
N<N_{\max }=\frac{1}{2} \frac{N_{\zeta} N_{\rho}^{2}}{\alpha / \Delta^{\prime}}
$$

where $N_{\rho}=L_{\rho} / \Delta_{\rho}$ and $N_{\zeta}=L_{\zeta} / \Delta_{\zeta}$ are the number of grid points along the $\rho$ and $\zeta$ directions, $L_{\rho}$ and $L_{\zeta}$ being the typical radial and axial size of the cylinder in length units, and $\Delta^{\prime} \equiv \Delta_{\rho}^{2} \Delta_{\zeta} / \Delta^{2}$ is an effective linear scale.

Since the scattering length $\alpha$ is typically much smaller than the characteristic radial length $N_{\rho} \Delta^{\prime}$, we conclude that the maximum time step is diffusion controlled, unless the number of bosons significantly exceeds the number of grid points in the simulation.

Before moving on to Sec. V, we observe that the present analysis is appropriate to the GPE as well, since the nonlinear term in this equation is local in configuration space. The nonlinearity may of course have a profound influence on the resulting physics, but as long as the stability criterion (12) is fulfilled, perhaps including a safety factor (see below), there is every reason to believe that this physics is correctly reproduced by the present numerical scheme. In order to secure a further margin of stability, we have adopted an empirical safety factor keeping the actual time step consistently below the marginal stability threshold, typically around 0.2 .

\section{NUMERICAL VALIDATION}

The numerical scheme described above has been validated in the following test cases: (i) ground state of the harmonic potential (no self-interaction), (ii) free self-interacting condensate (no external potential), (iii) free expansion of a self-interacting condensate, (iv) gravity-driven transport of a condensate in a cylindrical optical potential. In cases (i)-(iii) above we use the typical values $S_{l}=1 \mu \mathrm{m}$ and $S_{t}=2.9 \mathrm{~ms}$ for length and time units.

\section{A. Ground state of the harmonic potential}

In cylindrical coordinates $(\rho, \zeta)$, the ground state in a harmonic potential of the form

$$
V_{\mathrm{ext}}(\rho, \zeta)=\frac{1}{4}\left(\rho^{2}+\epsilon \zeta^{2}\right)
$$

is given by the following wave function:

$$
\Phi(\rho, \zeta ; \tau)=\rho \frac{\epsilon^{1 / 8}}{(2 \pi)^{3 / 4}} e^{\left(\rho^{2}+\epsilon \zeta^{2}\right) / 4} e^{-i[(2+\epsilon) / 2] \tau},
$$

with the normalization $2 \pi \int|\Phi|^{2} \rho^{-1} d \rho d \zeta=1$. It is therefore expected that by starting with Eq. (16) at $\tau=0$ as an initial condition, the numerical scheme would leave the condensate density basically unchanged as time unfolds.

This property has been tested on a $[0,5] \times[-5,5](\rho, \zeta)$ domain with $21 \times 21,41 \times 41$, and $81 \times 81$ grid points. In the two former cases, a time step $\Delta \tau=10^{-3}$ has been imposed, whereas in the latter case $\Delta \tau=0.5 \times 10^{-3}$. In all cases, the time span of the simulation covers $T=10$ temporal units. Visual inspection of the wave function does not reveal any appreciable change of its square modulus with time.

To back up visual inspection with quantitative data, we have also monitored the change in time of the global error $e$ defined as

$$
e^{2}(\tau)=\int\left|\Phi^{A}-\Phi^{N}\right|^{2} 2 \pi \rho^{-1} d \rho d \zeta
$$

where the superscripts $A$ and $N$ refer to the analytical and numerical solutions, respectively. The time evolution of the global error for the three grid resolutions is reported in Fig. 1.

From these curves we infer a quadratic error accumulation in time. However, the prefactor of the quadratic term is quite small and rapidly decreasing with grid resolution. From the present series at three different resolutions we infer a power decay $e^{2} \sim \Delta^{-2 p}$ with $p \sim 2$ (note that the square error is shown in Fig. 1).

The net result is that a moderate-resolution $81 \times 81$ grid already secures a global error below $10^{-5}$ over a signifi- 


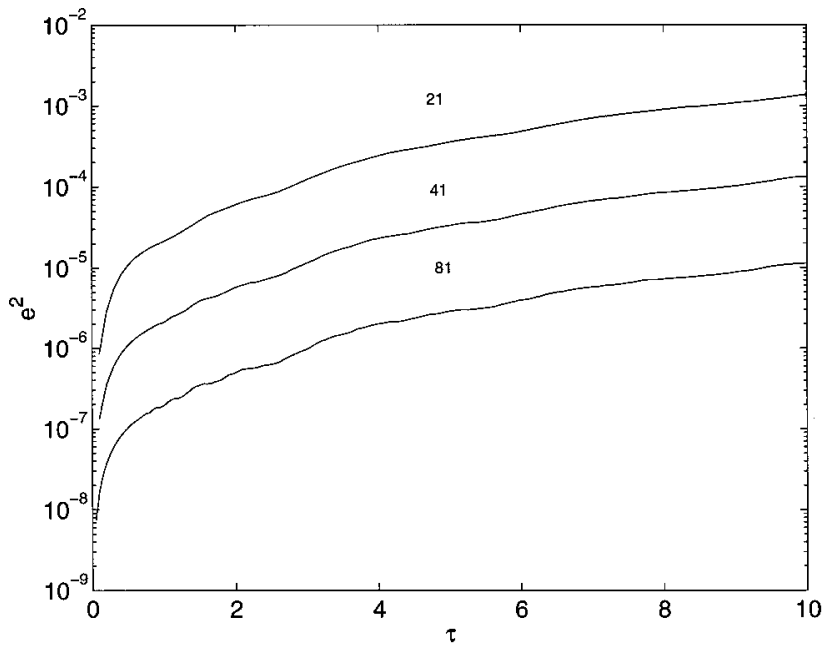

FIG. 1. Ground state of the harmonic potential. Square norm of the global error $e^{2}$ as a function of the dimensionless time $\tau$, as defined in Eq. (17). The three curves refer to three different grid resolutions $(21 \times 21,41 \times 41,81 \times 81)$.

cantly long period of time; that is, ten oscillation periods. A closer inspection of the separate contributions from the real and imaginary components of the wave function to the global error $e_{R}^{2}$ and $e_{I}^{2}\left(e^{2}=e_{R}^{2}+e_{I}^{2}+\right.$ interference terms $)$ reveals an interesting dip-tip anticorrelation: errors coming from the real part peak at the minima of the errors coming from the imaginary one and vice versa (see Fig. 2). The result is no interference and hence systematic, though very slow, accumulation in time.

\section{B. Free self-interacting condensate}

In the next series of tests we evaluate the evolution in time of a free self-interacting condensate (no external potential), starting from a numerically convenient analytic solution of the free GPE for the initial wave function $\Phi(\rho, \zeta ; \tau=0)$. The integration domain is $[0<\rho<1.24] \times[0.1<\zeta<0.3]$ and

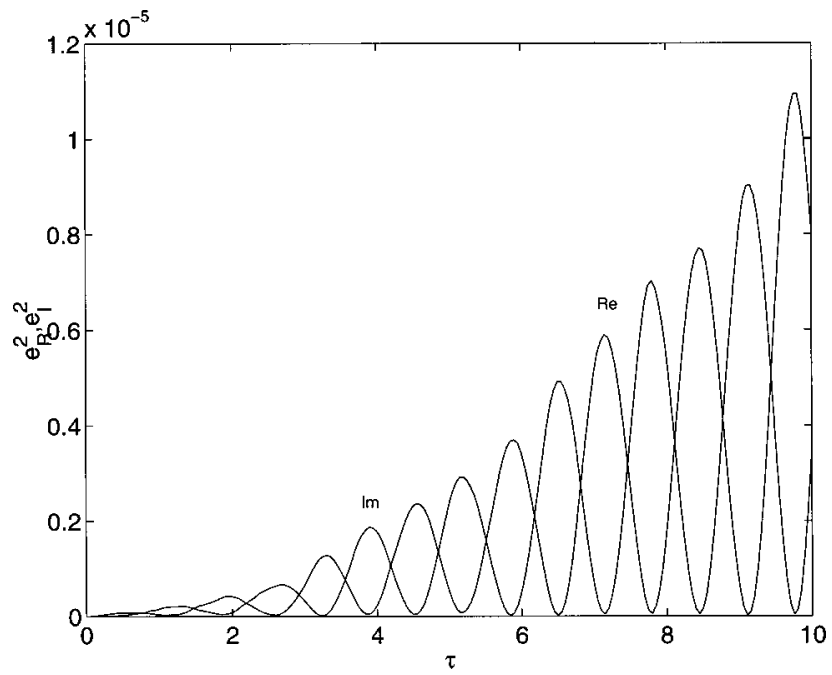

FIG. 2. Ground state of the harmonic potential. Square norm of the errors $e_{R}^{2}$ and $e_{I}^{2}$ associated with the real and imaginary parts of the wave function as functions of the dimensional time $\tau$, as defined in Eq. (17). The grid is $81 \times 81$.

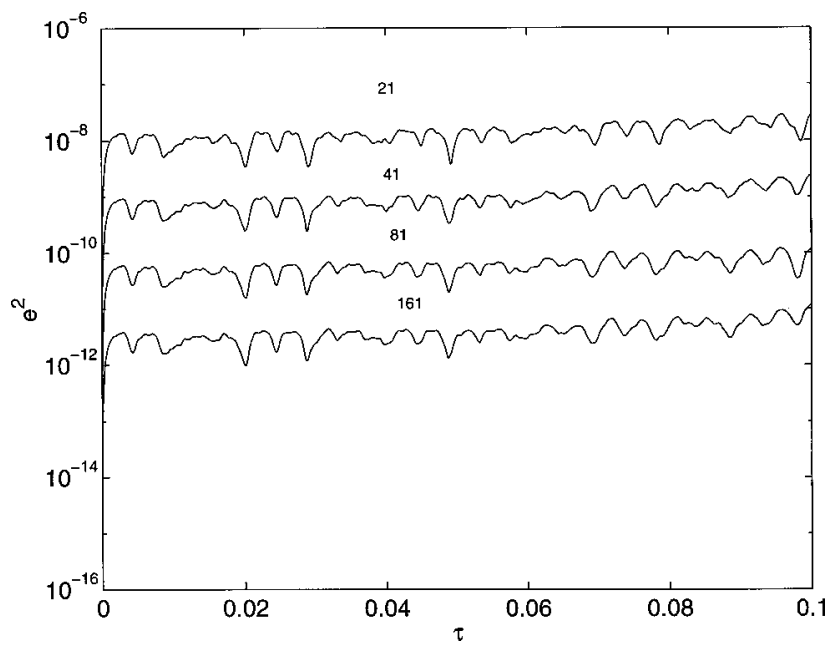

FIG. 3. Free self-interacting condensate. The results of the simulation are compared with the analytic form $\Phi(\rho, \zeta ; \tau)$ $=e^{-8 i \pi \alpha N \tau} \Phi(\rho, \zeta ; 0)$, where $\Phi(\rho, \zeta ; 0)=\rho\left(e^{\sqrt{16 \pi \alpha N \zeta}}+1\right)\left(e^{\sqrt{16 \pi \alpha N \zeta}}\right.$ $-1)^{-1}$ is merely a convenient solution of the free GPE for the purpose of testing the scheme in a time-dependent scenario. The figure shows the square norm of the global error (real plus imaginary part of the wave function) in the case $8 \pi \alpha N=210$, which corresponds to $a=110$ Bohr radii and $N=10^{4}$. The four curves refer to four different resolutions $(21 \times 21,41 \times 41,81 \times 81$, and $161 \times 161)$

the integration proceeds over $T=0.1$ time units, corresponding to about three oscillation periods. Four different resolutions, i.e., $21 \times 21,41 \times 41,81 \times 81$, and $161 \times 161$ have been adopted, the time step being $\Delta \tau=10^{-6}, 5 \times 10^{-7}, 10^{-7}$, and $5 \times 10^{-8}$, respectively.

In Fig. 3 we report the square norm of the error as a function of time, as defined by Eq. (17) using $\Phi(\rho, \zeta ; \tau)$ $=e^{-8 i \pi \alpha N \tau} \Phi(\rho, \zeta ; \tau=0)$ for the analytic wave function. From this figure, again, an excellent agreement with analytical results is obtained even on the very coarse $21 \times 21$ grid.

\section{Free expansion of a self-interacting condensate}

We now turn our attention from purely numerical tests to physical applications. We consider here the free expansion of a cylindrical self-interacting condensate that is initially confined in a harmonic trap. Such a situation is commonly realized in the experiments as one of the steps needed in probing the system by absorption imaging [1]. Numerical simulations as well as analytical time-dependent Ansätze are available $[8,18]$.

We start with an elongated condensate having $\epsilon=8$ and let it freely expand by suddenly switching off the trap at $\tau$ $=0^{+}$. The simulation refers to $N=4000{ }^{87} \mathrm{Rb}$ atoms. The numerical parameters are as follows: integration domain $[0$ $<\rho<40 \sqrt{2}] \times[-20<\zeta<20]$, grid $121 \times 121$. The typical time step is $\Delta \tau=10^{-5}$. Due to the large size of the computational domain, a nonuniform mesh along the radial coordinate has been adopted. Boundary conditions are periodic along $\zeta$ and Dirichlet-type $\Phi=0$ at $\rho=40 \sqrt{2}$. At $\rho=0$ the symmetry condition $d \Phi / d \rho=0$ is imposed.

For illustrative purposes, the initial condition was chosen in the form of an elongated Gaussian $\Phi(\rho, \zeta ; \tau)$ $=A e^{-\beta\left(\rho^{2}+\epsilon \zeta^{2}\right)}$ with $A$ a normalization constant and $\beta$ 

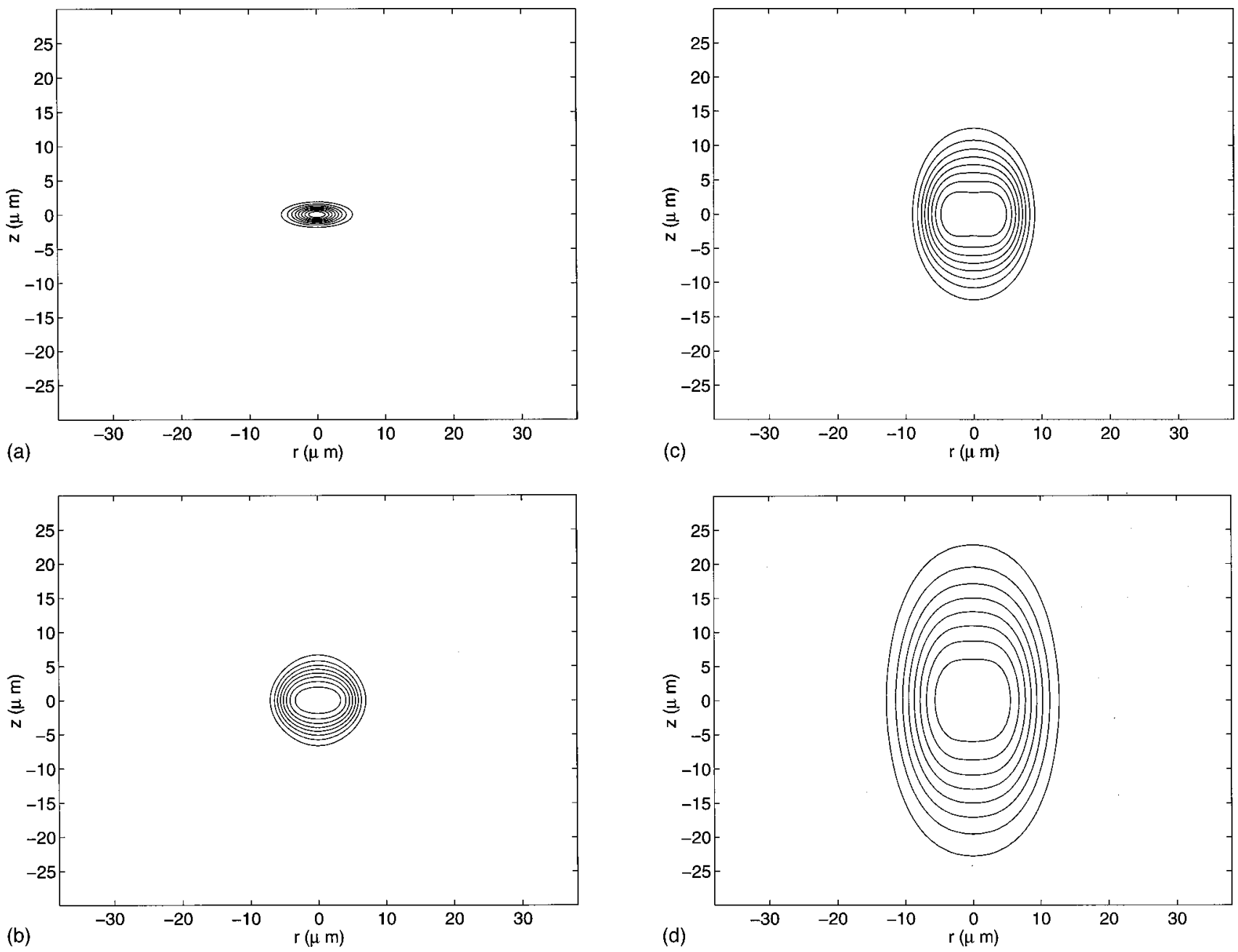

FIG. 4. Free expansion of a self-interacting condensate. (a)-(d) Contour plots of the condensate density at $t=0,4.4,8.8$, and $17 \mathrm{~ms}$. The grid is $121 \times 121$.

$=0.04$, which is close to the initial condition inferred from the experimental data of Fig. 3 in [8]. A more accurate initial condition would consist of an optimized matching between the Thomas-Fermi solution near the center of the cloud and a Gaussian tail [8].

Previous experimental and numerical data $[8,19]$ show that in the course of the expansion the condensate turns from a $\zeta$-elongated to a $\rho$-elongated shape. Such a behavior is reproduced by our numerical method, as witnessed by the results shown in the sequence of Figs. 4(a)-4(d). In Fig. 4(a) the contour lines of the initial condensate are shown, whereas Figs. 4(b)-4(d) show the same information at later times $t=4.4,8.8$, and $17 \mathrm{~ms}$, respectively.

Our data show good qualitative agreement with previous numerical and experimental results given in [8]. To quantify this statement we report in Fig. 5 the condensate widths along $\rho$ and $\zeta$, defined as $\sigma_{r}=\sqrt{\left\langle(r-\langle r\rangle)^{2}\right\rangle}$ and $\sigma_{z}$ $=\sqrt{\left\langle(z-\langle z\rangle)^{2}\right\rangle}$, where brackets denote space averaging $(\langle\rangle$ $\left.\equiv 2 \pi \int \ldots|\Psi|^{2} r d r d z\right)$.

Figure 5 shows that our results are basically consistent with the experimental data, although an underestimate (by $10-30 \%$ ) of the expansion rate appears in the long term. Better agreement with the data requires a more realistic model for the initial density profile, as used in the theoretical analysis made in Ref. [8].
In fact, as already mentioned, the evolution of the condensate is found to be rather sensitive to the initial conditions and especially to its width: thin condensates expand faster than thick ones and eventually develop double-humped profiles. In addition to more realistic choices of the initial conditions, the study of the dynamics of these thin condensates requires a substantial increase in space and time resolution, with a correspondingly higher demand of computational resources. Our actual results, ensuring norm conservation up to the fifth digit all along the simulation, are meant to represent a fair compromise between accuracy and efficiency.

\section{Gravity-driven transport in an optical potential}

The present numerical scheme has been successfully applied to the numerical simulation of a condensate in an effectively one-dimensional periodic potential [20]. The study of such a system is relevant in connection with the experiments by Anderson and Kasevich [11], in which a nearly pure condensate is poured from a magneto-optic trap into an optical lattice created by a detuned standing wave of light. Tunneling from well states to the continuum is driven by the gravitational field and interference between the condensates in different lattice sites manifests itself in the emission of falling drops. These are interpreted as coherent matter-wave 


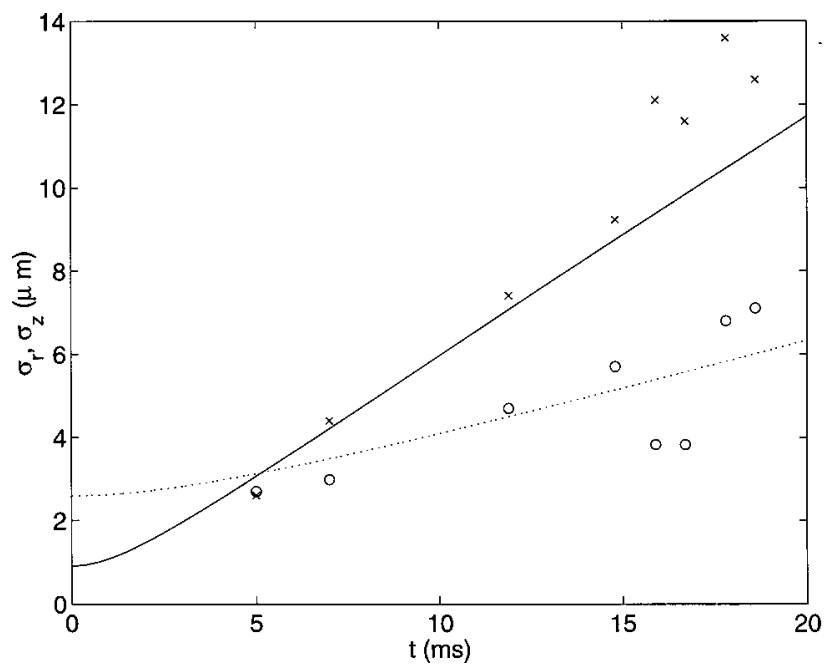

FIG. 5. Free expansion of a self-interacting condensate. Radial $\left(\sigma_{r}\right)$ and axial $\left(\sigma_{z}\right)$ widths of the condensate as functions of time. Solid line: axial, numerical. Dotted line: radial, numerical. Crosses: axial, experimental. Circles: radial, experimental.

pulses in analogy with a mode-locked photon laser [11]. The size of the drops is found to be $10 \%$ of the initial condensate size and the time interval between drops is estimated to be $1.1 \mathrm{~ms}$, in agreement with the expected period of Bloch oscillations.

The external potential, written in cylindrical coordinates $r$ and $z$, is $U_{\text {ext }}(r, z)=U_{l}(r, z)-m g z$, where the optical trap $U_{l}(r, z)$ can be modeled as $U_{l}(r, z)=U_{l}^{0} \exp \left(-r^{2} /\right.$ $\left.r_{l b}^{2}\right) \sin ^{2}(k z)$ and $g$ is the acceleration of gravity. $U_{l}^{0}$ is the well depth, scaling linearly with the intensity of the laser beam, $r_{l b}$ is the transverse size of the beam, and $k=2 \pi / \lambda$ is the laser light wave number, the lattice period being $\lambda / 2$.

Typical system parameters that are relevant to the experiment on ${ }^{87} \mathrm{Rb}$ [11] are $a=110 a_{0}$ with $a_{0}$ the Bohr radius, $N=10^{4}, \lambda=850 \mathrm{~nm}, r_{l b}=80 \mu \mathrm{m}, U_{l}^{0}=1.4 E_{R}$, with $E_{R}$ $=\hbar^{2} k^{2} / 2 m$ being the recoil energy. Finally, a number $n_{w}$ $\simeq 30$ wells are loaded initially.

The effect of the interactions can be studied in our simulations by varying the product $a N$ with a consistent change in the number $n_{w}$ of occupied wells, since $n_{w}$ depends on the strength of the interactions at given confinement parameters. The number of occupied wells is then given by the ratio of the size of the initial condensate to the size of each well, namely, $n_{w}=2 \zeta S_{l} / \lambda$ where $\zeta=\left(32 \pi N a / S_{l}\right)^{1 / 5}$ and $S_{l}$ $=\sqrt{\hbar / 2 m \omega}$ with $\omega$ the axial frequency of the magnetic trap.

In Ref. [20] the system was made effectively onedimensional (1D), using a result by Jackson et al. [21] to renormalize the scattering length. The reduction of the twodimensional (2D) GPE with cylindrical geometry to a 1D one was implemented by using an effective scattering length $\widetilde{a}=a \sqrt{U_{l}^{0}} /\left(r_{l b} \lambda\right)$. Within this model an extensive study of the emission, shape, and size of the pulses was given. Here we treat explicitly the transverse confinement by solving the full 2D problem in cylindrical symmetry.

We turn to discuss the choice of the initial value $\Psi(r, z ; t=0)$. The transverse part of the condensate wave function is taken as a Gaussian, with a width that is the harmonic approximation to the transverse shape of the optical potential. The corresponding frequency is $\omega_{r}$

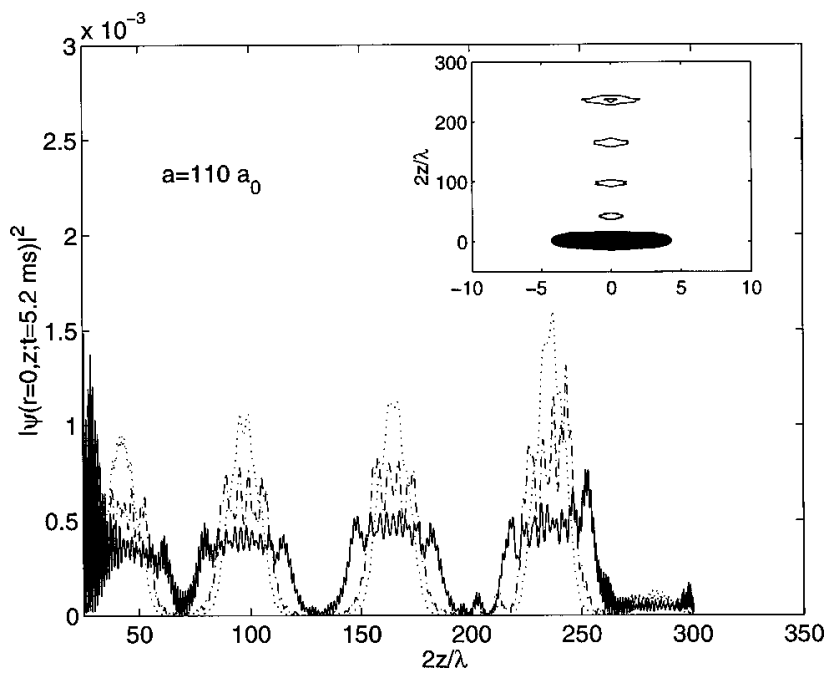

FIG. 6. Gravity-driven transport of a self-interacting condensate in a periodic potential. Density profile of the condensate after 5.2 $\mathrm{ms}$, as a function of $2 z / \lambda$ at $r=0(\lambda / 2$ is the spatial period $)$ in the interacting case $a=110 a_{0}$ and for $U_{l}^{0}=1.4 E_{R}$. For comparison the dotted curve reports the noninteracting case $a=0$. Dashed curve: $N=10^{4}$; solid curve: $N=10^{5}$. All other input parameters are listed in the text. Inset: the same as in the main body of the figure in the noninteracting case, but showing contour plots. The abscissa refers to the transverse distance in micrometers.

$=\sqrt{2 \alpha E_{R} /\left(m r_{l b}^{2}\right)}$, where $\alpha=U_{l}^{0} / E_{R}$. The overall shape of the density profile along the axial direction reflects instead the shape of the condensate inside the magnetic trap and is taken as a Gaussian with a width $\sqrt{\hbar / m \omega_{\text {eff }}}$ renormalized by the interactions, namely $\omega_{\text {eff }}=\left(4 \pi^{3 / 5} / \zeta^{2}\right) \omega$ [22]. Moreover, we assume that the lowest state of each well is occupied by a portion of condensate, whose Gaussian wave function is centered at the given site and is characterized by the frequency $\omega_{z}=2 \sqrt{\alpha} E_{R} / \hbar$.

A reasonable choice of the initial condition $\Psi(r, z ; t$ $=0$ ), taken to be centered at $r=0$ and $z=0$ then reads

$$
\Psi(r, z ; t=0)=A e^{-m \omega_{r} r^{2} / 2 \hbar} e^{-m \omega_{\mathrm{eff}} z^{2} / 2 \hbar} \sum_{l} e^{-m \omega_{z}(z-l \lambda / 2)^{2} / 2 \hbar} .
$$

Here, $A$ is a normalization factor and $l$ labels the occupied sites, their total number being $n_{\omega}$. In Eq. (18) we have assumed equal phases of the condensate at each site.

Let us discuss now the results of the simulation. We use a grid resolution of $21 \times 16$ on each single well, the time step being $\Delta \tau=2 \times 10^{-6}$. The main body of Fig. 6 shows three pictures of drop emission for different coupling strengths, by plotting the density profiles at $r=0$ as functions of $2 z / \lambda$, taken with $U_{l}^{0}=1.4 E_{R}$ after $5.2 \mathrm{~ms}$. The central condensate has been subtracted away. Contour plots showing the radial profiles are displayed in the inset. The dotted curve reports the behavior of the noninteracting gas, namely the case $a$ $=0$ and $n_{\omega}=31$. The dashed curve refers the interacting case with $a=110 a_{0}, N=10^{4}$, and $n_{\omega}=31$, while the solid curve shows the results for $a=110 a_{0}$ and $N=10^{5}$ with $n_{\omega}=49$. All the other parameters are as in the experiment (see above).

The main qualitative features of the drops are very similar to those resulting from the $1 \mathrm{D}$ simulation reported in [20]. 
We first note that each drop extends over a number of wells equal to that occupied by the initial condensate. Second, in all three cases the drops are equally spaced by 70 wells from center to center. This spacing corresponds to $1.1 \mathrm{~ms}$ of simulation time, in agreement with the measured value [11] and with the expected value of $1.09 \mathrm{~ms}$ for the period $T_{B}$ of Bloch oscillation $\left(T_{B}=4 \pi \hbar / m g \lambda\right.$, independently of the amplitude of the periodic potential and of the strength of the interactions). Third, we systematically find that both the width and the shape of each drop reproduces those of the parent condensate, giving proof of the coherent emission and suggesting a practical way to tailor matter-wave laser pulses.

Some differences between the 1D and the 2D simulations show up after a quantitative analysis of the number $N_{\text {drop }} / N$ of atoms per drop. We evaluate $N_{\text {drop }} / N \simeq 12 \%, 9 \%$, and $8 \%$ for the first three drops in all three cases (noninteracting, interacting with $N=10^{4}$, and interacting with $N=10^{5}$ ). These values are to be contrasted with the results of the $1 \mathrm{D}$ simulation, which give $N_{\text {drop }} / N$ of the same order, but increasing with increasing repulsive interaction strength.

Of course, the interactions tend to lift the bound state toward the continuum by an amount that may be measured by the the mean interaction energy per particle $E_{I}$. This is proportional to the product of the effective scattering length times the particle density and in the 1D simulation we have $E_{I} \propto \widetilde{a} / \lambda \propto a / \lambda^{2} r_{\mathrm{lb}}$. In the $2 \mathrm{D}$ case we have instead $E_{I}$ $\propto a / \lambda r_{\mathrm{lb}}^{2}$, which is significantly lower since $r_{\mathrm{lb}} \gg \lambda$. Namely, in the 2D simulation an increase in coupling strength is more readily compensated by a transverse spreading of the condensate and the value of $N_{\text {drop }} / N$ is insensitive to the interactions in the present range of system parameters.

We conclude by remarking that an increase in the coupling strength may eventually lead to disruption of the drops for two main reasons: (i) the bound state is lifted up to merge into the continuum, and (ii) the drops increase in size until they overlap with each other, their separation being determined solely by the lattice constant and by the slope of the external potential (i.e., by $\lambda$ and $g$ ). In the range of parameters of the experiment of Anderson and Kasevich [11], only the second mechanism may be significant.

\section{COMPUTATIONAL PERFORMANCE AND FUTURE PROSPECTS}

Having shown that the synchronous Visscher scheme provides a viable tool for the numerical investigation of BoseEinstein condensate dynamics, a few comments on computational performance as well as some comparative remarks with existing methods are in order. The present code takes about $10 \mathrm{~ms} /$ grid-point per step on a SUN-Ultra4 SPARC1 processor $(167 \mathrm{MHz})$, with no specific optimization effort. On a typical $100 \times 100$ grid with $\Delta \tau=10^{-5}$, a typical simulation lasting $T=10$ time units takes approximately $10^{4} \mathrm{CPU}$ seconds, namely, a few hours.

The key issue to performance is the size of the time step. Leaving aside very dense condensates with over tens of millions of atoms in a characteristic trap volume, the time step is limited by the spatial resolution. In fact, due to the explicit treatment of the diffusive kinetic-energy term, the time step $\Delta t$ scales approximately with the square of the size of the grid spacing $\Delta x$. This is a severe bound, which can be re- laxed by either moving to a semi-implicit time-marching scheme, or by adopting modern explicit schemes such as those mentioned below.

The main distinction between implicit versus explicit methods has been already addressed in the opening of this paper. Obviously, one would like to get the best of the two worlds: either a fast implicit method, or an unconditionally stable explicit method. As far as we can judge, the general trend (not just in BEC research) points rather to the latter alternative.

For instance, modern research in numerical fluid dynamics is moving in the direction of unconditionally stable explicit schemes based on clever matrix representations of the discrete evolution operator (transfer matrix) [23]. The idea is to decompose the transfer matrix from $t$ to $t+d t$ in such a way that each subsystem can be advanced analytically in time using exact exponential representations instead of firstor second-order polynomial expansions thereof.

On a similar vein, lattice kinetic methods based on the quantum generalization of the lattice Boltzmann scheme for fluid dynamics [24] have also been preliminarily applied to the evolution of one-dimensional BEC's [25]. These schemes are very fast, ideally suited to parallel computing, but unfortunately they do not extend straightforwardly to multidimensional non-Cartesian geometries.

Finally, it is interesting to notice that the same idea of looking for analytical expressions of short-time numerical propagators is also gaining popularity in the simulation of classical and quasiclassical condensed-matter systems using molecular dynamics techniques $[26,27]$. Here, the clever partitioning is reconduced to a Trotter representation of the evolution operator: $e^{i H \Delta t}=e^{i H_{1} \Delta t / 2} e^{i H_{2} \Delta t} e^{i H_{1} \Delta t / 2}$ where $H=H_{1}$ $+H_{2}$ is the total Hamiltonian, and $H_{1}, H_{2}$ is a pair of commuting operators typically associated with kinetic and potential energy. One of the main virtues of these schemes is that they lead to sympletic time integrators, i.e., time-marching schemes preserving the phase-space volume elements (Liouville theorem). This secures numerical reversibility, which is an excellent prerequisite for enhanced numerical stability. Since the synchronous Visscher method is naturally hinting at an ensemble of lattice walkers, whose collective motion describes the wave function dynamics, it would be interesting to explore whether the present work can be extended in the direction of producing unconditionally stable simpletic integrators for BEC dynamics.

\section{CONCLUSIONS}

In summary, we have presented the application of an explicit scheme for the numerical solution of a Schrödinger equation with local nonlinear interactions in twodimensional cylindrical geometry. The present results indicate that this explicit scheme provides a flexible and competitive tool for the numerical study of the dynamics of Bose-Einstein condensates. In particular, the case of BEC transport in optical potentials highlights the need for a timeaccurate description of droplet ejection from the main condensate and other fast dynamic events.

\section{APPENDIX: PROOF OF UNITARITY}

With the definition of discrete probability (10), we obtain (one-dimensional case for simplicity) 


$$
\begin{aligned}
\delta P \equiv & \sum_{j}\left(P_{j}^{k+1}-P_{j}^{k}\right) \\
= & \sum_{j} \operatorname{Re}_{j}^{k+1} \operatorname{Re}_{j}^{k}+\operatorname{Im}_{j}^{k+1} \operatorname{Im}_{j}^{k}-\operatorname{Re}_{j}^{k} \operatorname{Re}_{j}^{k-1} \\
& -\operatorname{Im}_{j}^{k} \operatorname{Im}_{j}^{k-1}
\end{aligned}
$$

which is

$$
\delta P=\sum_{j} \operatorname{Re}_{j}^{k}\left(\operatorname{Re}_{j}^{k+1}-\operatorname{Re}_{j}^{k-1}\right)-\operatorname{Im}_{j}^{k}\left(\operatorname{Im}_{j}^{k+1}-\operatorname{Im}_{j}^{k-1}\right) .
$$

Upon using the equations of motion (6), the above expression yields

$$
\delta P=\Delta \tau \sum_{j} \operatorname{Re}_{j}^{k}\left(\mathcal{H}_{j}^{k} \operatorname{Im}_{j}^{k}\right)-\operatorname{Im}_{j}^{k}\left(\mathcal{H}_{j}^{k} \operatorname{Re}_{j}^{k}\right),
$$

where $\mathcal{H}_{j}^{k} \equiv \mathcal{T}_{j}+\mathcal{V}_{j}^{k}$ is the total Hamiltonian, whose time dependence is entirely due to the nonlinear self-interaction potential. Since this self-interaction is local in space, it contributes zero to the above sum term-by-term, i.e., $\operatorname{Re}_{j}^{k} \mathcal{V}_{j}^{k} \operatorname{Im}_{j}^{k}$ $-\operatorname{Im}_{j}^{k} \mathcal{V}_{j}^{k} \operatorname{Re}_{j}^{k}=0$, which means that, like in the linear case, we are only left with surface contributions due to the kineticenergy operator.
Reinstating the spatial bounds for clarity and omitting the by now redundant temporal index $k$, a direct calculation yields

$$
\delta P=\frac{\delta \tau}{\delta \zeta^{2}} \sum_{j=0}^{J+1} \operatorname{Re}_{j}\left(\mathcal{T}_{j} \operatorname{Im}_{j}\right)-\operatorname{Im}_{j}\left(\mathcal{T}_{j} \operatorname{Re}_{j}\right)
$$

$$
\begin{aligned}
= & \operatorname{Re}_{1}\left(\operatorname{Im}_{0}-\operatorname{Im}_{1}\right)+\operatorname{Im}_{1}\left(\operatorname{Re}_{1}-\operatorname{Re}_{0}\right) \\
& +\operatorname{Re}_{J}\left(\operatorname{Im}_{J+1}-\operatorname{Im}_{J}\right)+\operatorname{Im}_{J}\left(\operatorname{Re}_{J}-\operatorname{Re}_{J+1}\right) .
\end{aligned}
$$

It is readily checked that all of these terms vanish identically under any of the three types of boundary conditions: (i) von Neumann (zero derivative at the boundary), $\Phi_{0}=\Phi_{1}$, $\Phi_{J}=\Phi_{J+1}$; (ii) Dirichlet (zero wave function at the boundary), $\Phi_{1}=0, \Phi_{J}=0$; periodic (same wave function on inlet/ outlet boundary), $\Phi_{1}=\Phi_{J}$ where $\Phi \equiv \operatorname{Re}, \mathrm{Im}$. The same analysis carries over to a two-dimensional cylindrical geometry, with only a bit of lengthier algebra.

\section{ACKNOWLEDGMENTS}

We thank Dr. M. Amoruso and Dr. A. Minguzzi for valuable discussions.
[1] M. H. Anderson, J. R. Ensher, M. R. Matthews, C. E. Wieman, and E. A. Cornell, Science 269, 198 (1995).

[2] C. C. Bradley, C. A. Sackett, J. J. Tollett, and R. Hulet, Phys. Rev. Lett. 75, 1687 (1995).

[3] K. B. Davis, M.-O. Mewes, M. R. Andrews, N. J. van Druten, D. S. Durfee, D. M. Kurn, and W. Ketterle, Phys. Rev. Lett. 75, 3969 (1995).

[4] See, e.g., Bose-Einstein Condensation, edited by A. Griffin, D. Snoke, and S. Stringari (Cambridge University Press, New York, 1995).

[5] For a recent review, see F. Dalfovo, S. Giorgini, L. P. Pitaevskii, and S. Stringari, Rev. Mod. Phys. 71, 463 (1999).

[6] L. D. Landau and E. M. Lifshitz, Non-Relativistic Quantum Theory (Pergamon, Oxford, 1960).

[7] M. J. Holland and J. Cooper, Phys. Rev. A 53, R1954 (1996).

[8] M. J. Holland, D. S. Jin, M. L. Chiofalo, and J. Cooper, Phys. Rev. Lett. 78, 3801 (1997).

[9] E. P. Gross, Nuovo Cimento 20, 451 (1961).

[10] L. P. Pitaevskii, Zh. Eksp. Teor. Phys. 40, 646 (1961) [Sov. Phys. JETP 13, 451 (1961)].

[11] B. P. Anderson and M. A. Kasevich, Science 281, 1686 (1998).

[12] M. Edwards, R. J. Dodd, C. W. Clark, P. A. Ruprecht, and K. Burnett, Phys. Rev. A 53, R1950 (1996).

[13] F. Dalfovo and S. Stringari, Phys. Rev. A 53, 247 (1996).

[14] P. Visscher, Comput. Phys. 596 (1991).

[15] R. Kosloff, J. Phys. Chem. 92, 2087 (1988); J. Chem. Phys. 68, 2794 (1978).
[16] A. R. Mitchell and D. F. Griffiths, The Finite Difference Method in Partial Differential Equations (Wiley, New York, 1980).

[17] D. Potter, Computational Physics (Wiley, London, 1977).

[18] Y. Castin and R. Dum, Phys. Rev. Lett. 77, 5315 (1996); F. Dalfovo, C. Minniti, S. Stringari, and L. P. Pitaevskii, Phys. Lett. A 227, 259 (1997).

[19] D. S. Jin, J. R. Enshen, M. R. Matthews, C. E. Wiemann, and E. A. Cornell, in Proceedings of the XXI International Conference on Low Temperature Physics [Czech. J. Phys. (to be published)].

[20] M. L. Chiofalo, S. Succi, and M. P. Tosi, Phys. Lett. A 260, 86 (1999).

[21] A. D. Jackson, M. Kavoulakis, and C. J. Pethick, Phys. Rev. A 58, 2417 (1998).

[22] G. Baym and C. J. Pethick, Phys. Rev. Lett. 76, 6 (1996).

[23] J. Richardson, R. Ferrell, and L. Long, J. Comput. Phys. 104, 69 (1993).

[24] S. Succi and R. Benzi, Physica D 69, 327 (1993).

[25] S. Succi, Phys. Rev. E 53, 1969 (1996); Int. J. Mod. Phys. C 9 , 1577 (1999).

[26] M. Tuckerman, B. Berne, and G. Martyna, J. Chem. Phys. 97, 1990 (1992).

[27] B. Berne, in Proceedings of the International School: Computer Simulations of Rare Events and the Dynamics of Classical and Quantum Systems, edited by B. Berne, J. Coker, and C. Ciccotti (World Scientific, Singapore, 1998), Chap VI, p. 131. 